\title{
Effect of Blue-Light-Emitting Diode Exposure on Collagen Density of Periodontal Ligament During Orthodontic Tooth Movement in Rats
}

\author{
Arifah Hariadi 1(D), Dyah Karunia 1(D), Christnawati Christnawati ${ }^{1}$ (D), Cendrawasih Andusyana \\ Farmasyanti ${ }^{1}$ (D), Ananto Ali Alhasyimi 1,* (D) \\ 1 Department of Orthodontics, Faculty of Dentistry, Universitas Gadjah Mada, Yogyakarta 55281, Indonesia; \\ arifah.hariadi@mail.ugm.ac.id (A.H.); dkarunia2008@yahoo.com $\quad$ (D.K.); $\quad$ christnawati@ugm.ac.id $\quad$ (C); \\ cendrawasih@ugm.ac.id (C.A.F.); anantoali@ugm.ac.id (A.A.A.); \\ * Correspondence: anantoali@ugm.ac.id (A.A.A.);
}

Scopus Author ID 57197808734

Received: 15.10.2021; Revised: 20.11.2021; Accepted: 24.11.2021; Published: 9.12.2021

\begin{abstract}
Photobiomodulation therapy using Light Emitting Diode (LED) with a certain period and intensity can stimulate tissue repair and accelerate orthodontic tooth movement. During remodeling, collagen on periodontal ligaments was formed on the pressure and tension sides. The aim of this study was to investigate the effect of exposure time and observation day of blue LED on the collagen density of periodontal ligament of the pressure and tension sides of Wistar rats. Forty-eight male Wistar rats aged 3-4 months weighing 300-500 grams were divided into four groups: the control group; the group exposed to LED with $\lambda 1000 \mathrm{~nm}$ of $490 \mathrm{~mW} / \mathrm{cm}^{2}$ for 25,30 , and 35 seconds once a day. In addition, their mandibular inter-incisor was administered with thirty-five grams orthodontic force using an open coil spring. The calculations on the collagen density of pressure and tension sides were carried out on days $0,3,7$, and 14 using the TinEye application. Statistical analysis test was conducted using two-way ANOVA and LSD post hoc test. The results showed a more significant increase in the collagen density of the pressure and tension sides than that of the control group ( $\mathrm{p}<0.05$ ). The collagen density of pressure and tension sides reach the highest point at 30 seconds exposure followed by 35 and 25 seconds. The collagen density of pressure sides decreased on the $3^{\text {rd }}$ day and increased afterward, and the tension sides continued to increase on the $3^{\text {rd }}$ day afterward. The study showed that blue light LEDs were able to increase collagen density on the pressure and tension sides, with optimal exposure at 30 seconds, and it reached the highest point at day 14 .
\end{abstract}

Keywords: Blue LED; photobiomodulation; exposure time; observation time; collagen density.

(C) 2021 by the authors. This article is an open-access article distributed under the terms and conditions of the Creative Commons Attribution (CC BY) license (https://creativecommons.org/licenses/by/4.0/).

\section{Introduction}

Orthodontic treatment is the process of achieving proper bite function and maximum aesthetics by moving the teeth through the application of physical force. Orthodontic treatment takes 1-3 years on average to get the results. Complications within orthodontic therapy, such as cavities, white spot lesion, periodontitis, gingivitis, and root resorption, are common among orthodontic appliance users during the extended treatment term [1,2]. Because of the negative effects of relatively long-term orthodontic therapy make efforts to accelerate orthodontic tooth movements necessary [3]. Photobiomodulation effect on Low-Level Laser (LLL) and Light Emitting Diode (LED) can be used to achieve efficient tooth movement. In addition, LEDs can be applied to a larger area of the body safely. LEDs in vivo experiments show that 
photobiomodulation plays a key role in inflammation by significantly stimulating angiogenesis and reducing tissue necrosis [4]. A periodontal ligament is a soft tissue that supports teeth that function to maintain the teeth' stability and elasticity along with interstitial fluid as a shock breaker during orthodontic tooth movement. Most of the components of the periodontal ligaments are collagen [5]. With orthodontic force, the periodontal ligament on the pressure and tension side form a new periodontal ligament with an increasing number of fibroblasts. Fibroblasts are cells that function to synthesize collagen matrix [6]. The increasing thickness of the epidermis and the number of fibroblasts and collagen in the wound are the indicators of wound healing initiation and tissue regeneration. The thicker and denser the collagen, the wound will be healing faster [7].

Ekizer's experiment uses LED with $\lambda 618 \mathrm{~nm}$ and $20 \mathrm{~mW} / \mathrm{cm} 2$ for 20 minutes to accelerate orthodontic tooth movement. However, twenty minutes is quite long for using the clinic [8]. LED with an $\lambda 1000 \mathrm{~nm}$ of $490 \mathrm{~mW} / \mathrm{cm}^{2}$ is commonly used in clinics as a composite hardener. Based on the conversion with the Arndt-Schulz Law, it was known that 30 seconds of LED exposure with 5 seconds range were assumed to increase the acceleration of orthodontic tooth movement. Armellin's research proved that the addition of 20 seconds increased the pulp chamber temperature by $0.5^{\circ} \mathrm{C}$ [9]. This study aims to analyze the effect of LED blue light exposure on the collagen density during orthodontic tooth movement on the pressure and tension side. The hypothesis of this study was that the exposure of LED increases collagen density on the pressure and tension side of the periodontal ligament.

\section{Materials and Methods}

Ethical clearance of this study was obtained from the Ethical Commission of Dentistry, Faculty of Dentistry, Gadjah Mada University number 00291/KKEP/FKG-UGM/EC/2020. Forty-eight Wistar male rats weighing $300 \mathrm{~g}$ to $500 \mathrm{~g}$ were used in this current study, which was chosen utilizing simple random sampling into four groups ( $\mathrm{n}=12 /$ group): control groups that were not exposed to LED; the grouped exposed to LED with $\lambda 1000 \mathrm{~nm}$ of $490 \mathrm{~mW} / \mathrm{cm} 2$ for 25,30 , and 35 seconds once a day. The samples were then divided into smaller groups based on the day of observation (day 0, 3, 7, and 14 after force delivery) ( $n=3$ ). Sample size $(n=3)$ was obtained utilizing Lemeshow's formula. A sample size of three animals in each group would give more than $80 \%$ power to detect significance. The animals were housed for a week before the experiment with a 12-hour light/dark cycle, a constant room temperature of $26^{\circ} \mathrm{C}$, and a humidity of 50 percent to allow them to acclimatize and compensate for their different origins before the experiment began. All animals were fed a conventional pellet diet (expanded pellets; Stepfield, UK) with access to reverse osmosis water ad libitum.
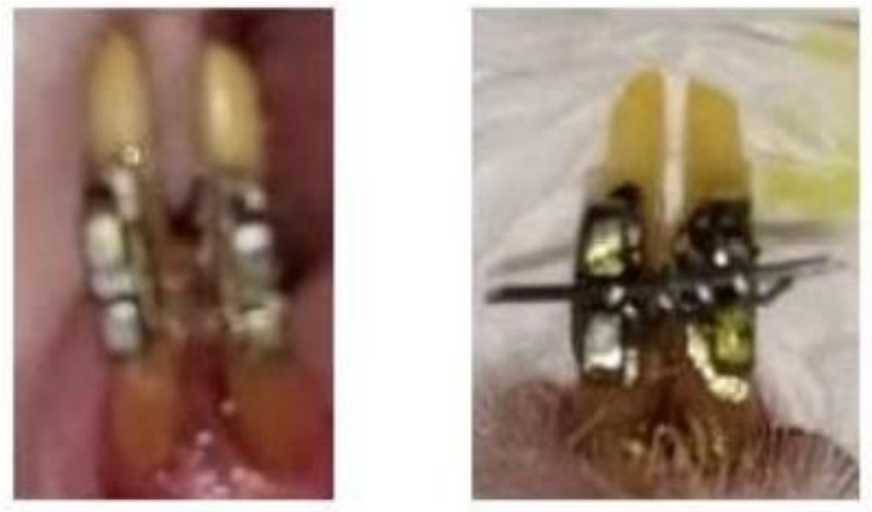

Figure 1. Cleat placement on Wistar rat mandibular incisor. 
Orthodontic appliance placement was done by anesthetizing animal models using 50 $\mathrm{mg} / \mathrm{kg}$ of ketamine and $5 \mathrm{mg} / \mathrm{kg}$ of xylazine by firstly using the intramuscular method and subsequently followed by etching and bonding the bracket of Edgewise standard slot 0,022" (American Orthodontics $®$, USA) that were cut in two pieces to adjust with the lower incisor width. Then, a rounded stainless steel wire with a diameter of 0.016 "(Ortho Organizer®, USA) was placed inside an open coil spring (NiTi 0,010" x 0.030", American Orthodontic ${ }^{\circ}$, USA) with $35 \mathrm{~g}$ force (Figure 1).

\subsection{LED exposure.}

Blue LED duration was determined based on the conversion from Ekizer [8] research

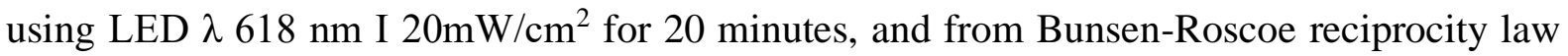
[10] with the long-duration low-intensity photon that equals to short-duration high-intensity photon. Photobiomodulation study using LED $\lambda 618 \mathrm{~nm} \mathrm{I} 20 \mathrm{~mW} / \mathrm{cm}^{2}$ in 20 minutes was proven to accelerate orthodontic tooth movement and increase mini-implant stability [8]. Thus, the conversion obtained optimal duration of LED exposure of $\lambda 490 \mathrm{~nm} \mathrm{I} 1000 \mathrm{~mW} / \mathrm{cm}^{2}$ of 30 seconds [11]. The LED exposure was administered once a day on the median line of lower incisors with a distance of $5 \mathrm{~mm}$ from the gingiva.

\subsection{Decapitation of the rats.}

The animals were euthanized using ketamine of $250 \mathrm{mg} / \mathrm{kg} \mathrm{BW}$, xylazine of $50 \mathrm{mg} / \mathrm{kg}$ BW intravenous, followed by alveolar bone dissection throughout the distal mesial side of the right and left mandibular incisors and apex. Tooth and mandibular specimens were collected on day 0 (baseline) and at the end of each treatment on days 3, 7, and 14. The tissue pieces were washed with saline solution and immersed in $4 \%$ formaldehyde fixation solution for 12 hours at $4^{\circ} \mathrm{C}$. The samples were decalcified using $10 \%$ EDTA-2Na solution at room temperature for 10 weeks. The specimens were subjected to graded alcohol dehydration: pure xylol at $4{ }^{\circ} \mathrm{C}$ and room temperature xylol. The samples were subsequently planted in paraffin block and cut in parallel to the long axis of the tooth in the mesiodistal direction of $5 \mu \mathrm{m}$. Histochemical staining was performed after deparaffinization.

\subsection{Collagen staining.}

Mallory's staining was carried out at the Anatomical Pathology Laboratory of the Faculty of Public Health Medicine and Nursing UGM. The slides that have been immersed in tissue staining (maximum 15 slides) were given $75 \mathrm{ml}$ of hematoxylin solution for 3-5 minutes. Afterward, the hematoxylin solution was poured into a storage bottle (can be reused up to 100 slides), then it was washed with distilled water 3 times for 1 minute. Each time the distilled water was washed, it was thrown away. Acid alcohol was put into the tissue, staining for 30 seconds, then the solution was poured into a storage bottle (50 slides can be used), and it was washed under running water for 3-5 minutes and rinsed with distilled water 1 time. Specimens were provided with $1 \%$ eosin solution for 1-2 minutes. The solution was poured into a storage bottle ( 50 slides can be used), then rinsed with distilled water 3 times. The specimens were subjected to dehydration and mounting, and the stained specimen was ready for observation. 


\subsection{Collagen density observation.}

Observation of the collagen density of periodontal ligament in the pressure and tension area of Wistar Rats' mandibular incisors was carried out on day 0, day 3, day 7, and day 14 . Observation of histological preparations was carried out using a binocular microscope with an objective lens of 400x magnification. Collagen was on the cementum surface in the compressed and stretched area of the periodontal ligament and was colored blue with Mallory staining. Every 1 slide and 5 visual fields were taken in the pressure area and 5 visual fields in the tension area of the periodontal ligament of the incisors of Wistar rats' incisors. Each field of view slide was photographed and converted into two colors digitally, namely blue and non-blue (Figure 8), using the TinEye application. The application calculated the percentage of blue and white color proportion. The percentage of collagen density was averaged from five visual fields of pressure and tension sides. The yield of each side preparation $(a \%+b \%+c \%+d \%+e \%)$ was divided by 5 . The result of the percentage calculation is presented in the form of quantitative data. The experiment resulted in normal and homogenous data. A two-way ANOVA parametric test was done to determine the difference between the exposure time group and the observation period, followed by Post Hoc (LSD) test. This study used a level of confidence of $95 \%(\alpha=$ $0.05)$.

\section{Results and Discussion}

\subsection{Pressure side collagen density.}

The mean and standard deviation values of collagen density on pressure side days 0,3 , 7, and 14 are shown in Table 1. The result of the two-way ANOVA test is depicted in Table 2. Collagen density on all LED exposure groups was significantly higher on the 4 subsequent observation days $(\mathrm{p}<0.05)$ than the result of the control group.

Table 1. Mean values and standard deviation (SD) of collagen density in periodontal ligament pressure areas in orthodontic tooth movement of Wistar rats groups CT, LT 25, LT30, and LT35.

\begin{tabular}{c|c|c|c|c}
\multirow{2}{*}{ Group } & \multicolumn{4}{|c}{ Mean values \pm Standard Deviation (SD) } \\
\cline { 2 - 5 } & (Day 0) & $($ Day 3) & $($ Day 7) & $($ Day 14) \\
\hline CT & $37.51 \pm 0.71$ & $28.13 \pm 0.15$ & $42.60 \pm 0.81$ & $62.30 \pm 1.04$ \\
\hline LT25 & $45.10 \pm 0.62$ & $36.30 \pm 0.79$ & $53.00 \pm 0.87$ & $65.30 \pm 0.53$ \\
\hline LT30 & $55.90 \pm 0.60$ & $52.40 \pm 1.34$ & $59.10 \pm 0.78$ & $74.30 \pm 1.21$ \\
\hline LT35 & $50.30 \pm 1.17$ & $42.10 \pm 1.08$ & $55.10 \pm 1.27$ & $67.60 \pm 1.05$
\end{tabular}

CT: control group (no exposure); LT25: 25 seconds LED exposure group; LT30: 30 seconds LED exposure group; LT35: 35 seconds LED exposure group.

Table 2. Two-way ANOVA test results the effect of exposure time and blue light LED observation day on the collagen density of periodontal ligament pressure areas on the orthodontic tooth movement of Wistar rats.

\begin{tabular}{c|c|c} 
Variable & $\mathbf{F}$ & p-value \\
\hline LED exposure time & 761.772 & $0.000^{*}$ \\
\hline Observation day & 1883.035 & $0.000^{*}$ \\
\hline $\begin{array}{c}\text { LED exposure time * Observation } \\
\text { day } \quad \text { Significant differences between groups }(p<0.05) .\end{array}$ & $0.000 *$
\end{tabular}

The collagen density on the pressure side in the exposure group with 25 seconds was higher than that of the control group. The group exposed to 30 seconds had the highest collagen density. The collagen density on the pressure side decreased on day 3 and increased on days 7 and 14 (Figure 2) and was statistically significant as tested by the Two-Way Anava test (Table 
2). Similarly, the Post Hoc test for all exposure and observation time groups was significant (Tables 3, 4) $(\mathrm{p}<0.05)$.

Table 3. Post Hoc LSD Test results between the length of exposure to collagen density in periodontal ligament pressure areas on the orthodontic tooth movement of Wistar rats.

\begin{tabular}{c|c|c|c|c} 
Group & CT & LT25 & LT30 & LT35 \\
\hline CT & & $-7.2892^{*}$ & $-17.7892^{*}$ & $-11.1393^{*}$ \\
\hline LT25 & $7.2892^{*}$ & & $-10.5000^{*}$ & $-3.8500^{*}$ \\
\hline LT30 & $17.7892^{*}$ & $10.5000^{*}$ & & $6.6500^{*}$ \\
\hline LT35 & $11.1393^{*}$ & $3.8500^{*}$ & $-6.6500^{*}$ &
\end{tabular}

Significant differences between groups $(p<0.05)$.

Table 4. The results of the Post Hoc LSD test between time observations of the collagen density in the periodontal ligament pressure area on orthodontic tooth movement of Wistar rats.

\begin{tabular}{|c|c|c|c|c|}
\hline Group & Day 0 & Day 3 & Day 7 & Day 14 \\
\hline Day 0 & & $7.4692 *$ & $-5.2475^{*}$ & $-20.1725^{*}$ \\
\hline Day 3 & $-7.4692 *$ & & $-12.7167 *$ & $-27.6417^{*}$ \\
\hline Day 7 & $20.1725^{*}$ & $5.2475^{*}$ & & $-14.9250^{*}$ \\
\hline Day 14 & $20.1725^{*}$ & $27.6417 *$ & $14.9250 *$ & \\
\hline
\end{tabular}

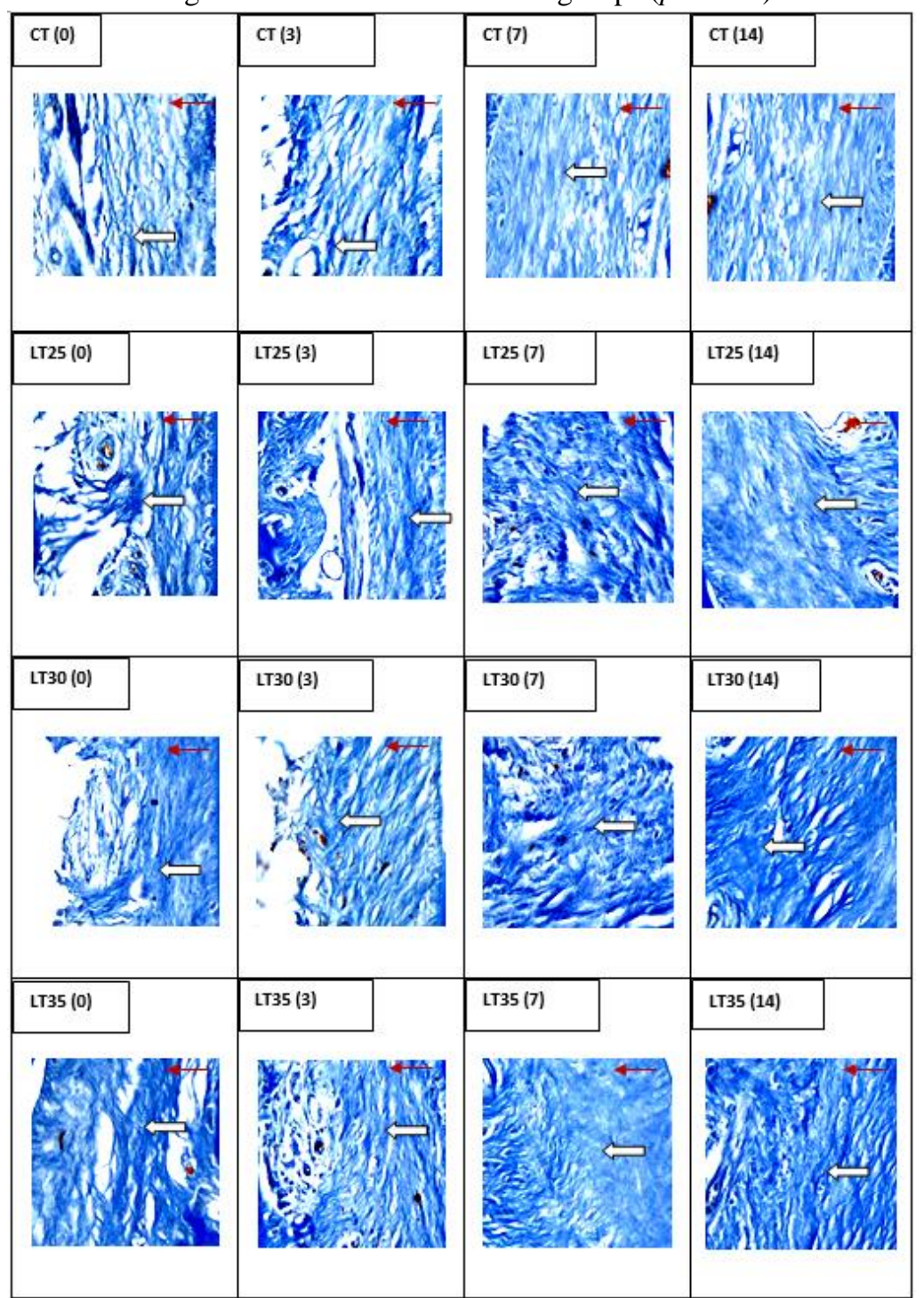

Figure 2. The difference in collagen density in the pressure side; CT $=$ No exposure; LT25 $=25$ seconds of exposure; LT30 $=30$ seconds of exposure; LT35 $=35$ seconds of exposure; $0=0$ day of exposure; $3=3$ rd day of exposure; $7=7$ th day of exposure; $14=$ Day 14 after exposure by Mallory painting at $400 x$ magnification;

Collagen is indicated by a white arrow, while the direction of the force is indicated by the red arrow. 


\subsection{Tension side collagen density.}

The mean and standard deviation values of collagen density on the tension side on days $0,3,7$, and 14 are shown in Table 5. The results of the two-way ANOVA test can be seen in Table 6.

Table 5. Mean values and standard deviation (SD) of collagen density in periodontal ligament tension areas in orthodontic tooth movement of Wistar rats groups CR, LR 25, LR30, and LR35.

\begin{tabular}{c|c|c|c|c}
\multirow{2}{*}{ Group } & \multicolumn{4}{|c}{ Mean values \pm Standard Deviation (SD) } \\
\cline { 2 - 5 } & (Day 0) & (Day 3) & (Day 7) & (Day 14) \\
\hline CR & $43.00 \pm 1.70$ & $48.40 \pm 0.62$ & $52.70 \pm 0.81$ & $64.50 \pm 0.80$ \\
\hline LR25 & $44.70 \pm 0.50$ & $53.90 \pm 1.40$ & $63.30 \pm 0.43$ & $72.60 \pm 0.70$ \\
\hline LR30 & $54.70 \pm 0.80$ & $65.60 \pm 1.13$ & $74.50 \pm 0.88$ & $84.80 \pm 1.15$ \\
\hline LR35 & $50.40 \pm 1.05$ & $58.40 \pm 0.65$ & $68.70 \pm 1.22$ & $78.50 \pm 0.52$
\end{tabular}

CR: control group (no exposure); LR25: 25 seconds exposure group; LR30: 30 seconds exposure group; LR35:

35 seconds exposure group.

Table 6. Two-way ANOVA test results the effect of exposure time and blue light LED observation day on the collagen density of periodontal ligament tension areas on the orthodontic tooth movement of Wistar rats.

\begin{tabular}{c|c|c} 
Variable & F & p-value \\
\hline LED exposure time & 739.543 & $0.000^{*}$ \\
\hline Observation day & 1704.475 & $0.000^{*}$ \\
\hline $\begin{array}{c}\text { LED exposure time * Observation } \\
\text { day }\end{array}$ & 14.016 & $0.000 *$ \\
*Significant differences between groups $(p<0.05)$. &
\end{tabular}

Collagen density in the tension side on the 35-second exposure group was lower than the results of the 30-second group but higher than those of the 25 seconds, and each exposure group was statistically significant as tested by the Two-Way Anava test and Post Hoc test ( $\mathrm{p}$ $<0.05)$. The collagen density on the tension side continued to increase on days 3, 7, and 14 (Figure 3) and was statistically significant as tested by the Two-Way Anava test (Table 6). Similarly, the Post Hoc test for all exposure and observation time groups was also significant (Tables 6 and 7$)(\mathrm{p}<0.05)$.

Table 7. Post Hoc LSD test results between the length of exposure to collagen density in periodontal ligament tension areas on the orthodontic tooth movement of Wistar rats

\begin{tabular}{c|c|c|c|c} 
Group & Control & $\mathbf{2 5}$ secs & 30 secs & 35 secs \\
\hline Control & & $-6.4750^{*}$ & $-17.7500^{*}$ & $-11.8500^{*}$ \\
\hline 25 secs & $6.4750^{*}$ & & $-112750^{*}$ & $-5.3750^{*}$ \\
\hline 30 secs & $17.7500^{*}$ & $11.2750^{*}$ & & $5.9000^{*}$ \\
\hline 35 secs & $11.8500^{*}$ & $\begin{array}{c}5.3750^{*} \\
\text { *Significant differences between groups }(p<0.05) .\end{array}$
\end{tabular}

Table 8. The results of the Post Hoc LSD test between time observations of the collagen density in the periodontal ligament tension area on orthodontic tooth movement of Wistar rats.

\begin{tabular}{|c|c|c|c|c|}
\hline Group & Control & Day 3 & Day 7 & Day 14 \\
\hline Control & & $-8.3750 *$ & $-16.6000^{*}$ & $-26.9000^{*}$ \\
\hline Day 3 & $8.3750 *$ & & $-8.2250 *$ & $-18.5250^{*}$ \\
\hline Day 7 & $16.6000^{*}$ & $8.2250^{*}$ & & $-10.3000^{*}$ \\
\hline Day 14 & $-26.9000 *$ & $18.5250^{*}$ & $10.3000^{*}$ & \\
\hline
\end{tabular}

The collagen density in the pressure area of the exposure group was higher than the control group on all observation days. LED (Light Emitting Diode) is a light that has a photobiomodulation effect that can stimulate cellular level activation. Cytochrome $\mathrm{C}$ oxidase 
(Cox) functions as the main light receptor and modifier for light-stimulating signals in cells. Cytochrome $\mathrm{C}$ oxidase is a terminal enzyme in the electron transport chain that can mediate the transfer of electrons from cytochrome $\mathrm{C}$ to oxygen molecules [12].

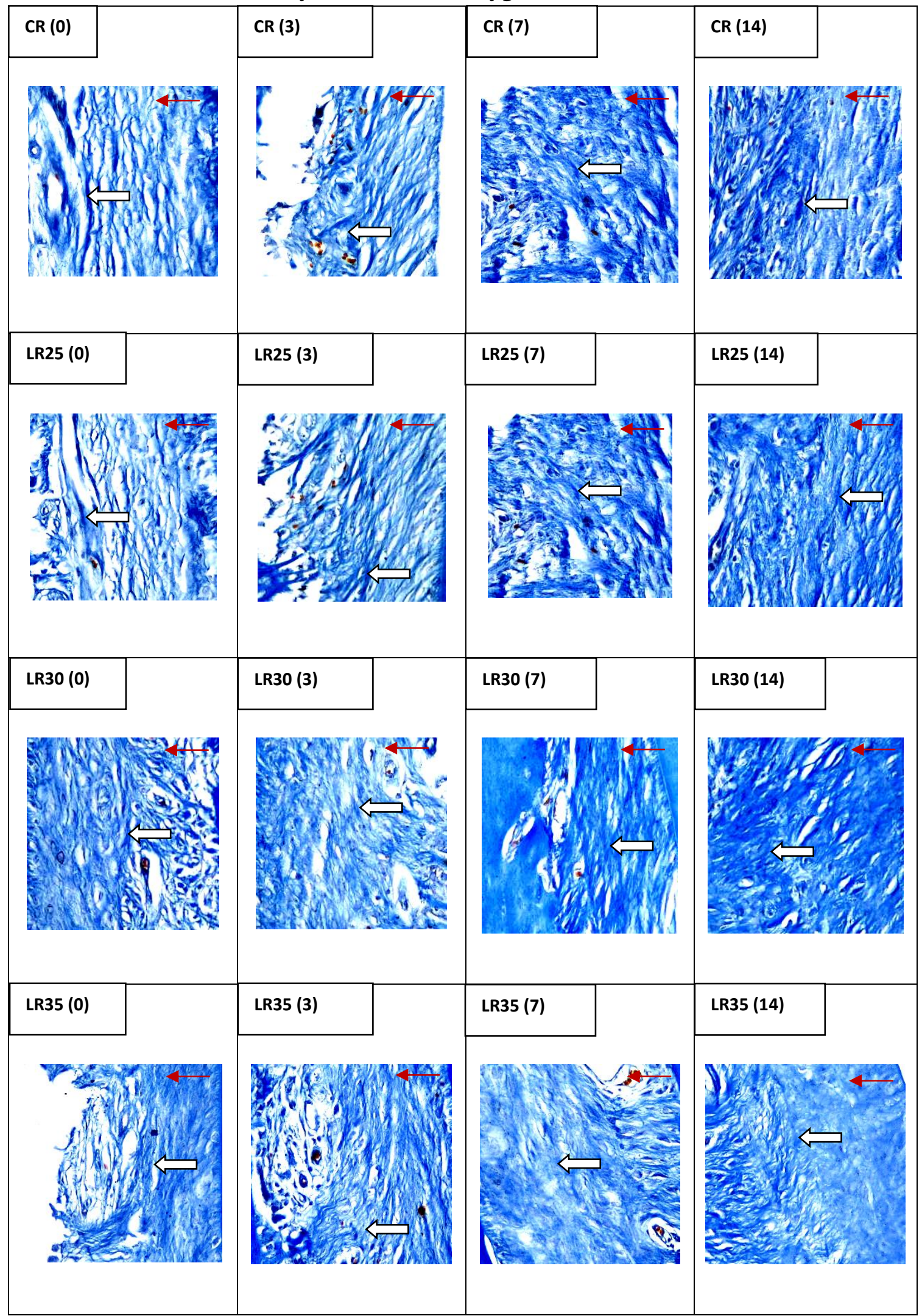

Figure 3. The difference in collagen density of the tension side; $C R=$ No exposure; LR $25=25$ seconds of exposure; LR30 = 30 seconds of exposure; LR35 = 35 seconds of exposure; $0=0$ day post exposure; $3=3 \mathrm{rd}$ day of exposure; $7=7$ th day of exposure; $14=$ Day 14 of exposure with Mallory painting at 400x magnification; Collagen is indicated by a white arrow, while the direction of the force is indicated by the red arrow. 
Photobiomodulation increases electrons for molecular oxygen reduction in the catalytic center of Cox. It heightens the potential for ATP, cyclic Adenosine MonoPhosphate (cAMP), Reactive Oxygen Species (ROS), Nitric Oxide (NO), NF-кB (Factor Nuclear Factor Kappa B), and mitochondrial membrane (MMP) [5,13-14, 15].

It has been shown that low-level light irradiation can change the levels of a wide range of cytokines and inflammatory mediators independent of whether they have pro- or antiinflammatory effects, such as TNF and other interleukins, histamine, TGF-beta, prostaglandins, and eicosanoids, among others. While it appears that photobiomodulation has an anti-inflammatory effect in the presence of inflammation, it appears that in the absence of inflammation, photobiomodulation produces pro-inflammatory mediators that could aid in tissue remodeling and modulate cell activity.[10]. Orthodontic force on the pressure side leads to inflammation; thereby, it can be understood that photobiomodulation increases collagen density due to Prostaglandin inhibition. Research conducted by Lim and colleagues has discovered that exposure to low-power $635 \mathrm{~nm}$ light can have an anti-inflammatory effect by reducing prostaglandin E2 (PGE2) production and cyclooxygenase 1 and 2 (COX-1 and COX2) gene transcription. [9]. The highest collagen density was found on day 14, which indicates that it took 14 times of LED exposure to get the highest collagen as much as $67 \%$. Further research needs to investigate whether more than 14 times exposure is required or not. In Ekizer's study, the exposure was done for 21 days once a day [8].

The duration of blue Light Emitting Diode for 30 seconds reached the highest collagen density in the periodontal ligament on the pressure area, followed by 35 seconds and 25 seconds in orthodontic tooth movement. This is in accordance with De Lima [16], who said that low doses with long exposure are equal to exposure to high-intensity rays in a short time, while exposure to weak doses slightly accelerates cell activity. In addition, a slightly stronger (optimal) exposure increases activity to peak, and eventually, a stronger exposure will have a negative effect on optimal exposure [17].

Based on the observation day, collagen density in the pressure area on the $3^{\text {rd }}$ day decreased because the periodontal ligament was pinched by the moving teeth due to orthodontic stress, leading to the decrease in collagen density. The stress which was applied to the periodontal ligament will cause hypoxia and decrease the blood supply. Consequently, the macrophages will phagocyte the dead tissue, resulting in a decrease in the number of periodontal ligament structures followed by removal of the alveolar bone. As the healing process occurs, new collagen will form in the pressure area to fill the resorbed alveolar bone $[18,19]$. Tooth movement takes 7-14 days after being exposed to orthodontic pressure. This trend is the same as the exposure group, which had higher collagen than the control group in all observation days.

The same trend also occurs on the tension side. The only difference was that the collagen density on the tension sides showed that the LED exposure group for 25, 30, and 35 seconds was higher on day 3 than the control group. Then, it increased gradually from day 7 to day 14, proving that observation affected collagen density on tension sides. The periodontal ligament in the tension area will remodel continuously with collagen, which will fill the tension area to become a new periodontal ligament. There is an increase in cell replication in the tension area because it is stimulated by stretching collagen fibers' periodontal ligament. Subsequently, the collagen fibers will continuously proliferate in the periodontal connective tissue until they fill the empty area to become a new periodontal ligament [2]. Afterward, the collagen density slowly increases starting from day 3 and continues to increase on day 7 to day 14 . This trend 
is different from that of the pressure, in which the collagen density decreased on day 3 and increased afterward. Collagen is formed from fibroblast cells, which play a role in bone remodeling and revitalization of periodontal ligament fibers for the stability of orthodontic treatment [20]. Tooth movement occurs simultaneously with the remodeling of active periodontal ligament collagen by new fibroblasts and deposition of new bone [21,22], increasing cell replication due to stimulation by the stretched periodontal ligament fiber bundles and the increase of blood vascularization [10].

One limitation of this study was the limited number of time points for evaluating restrictions throughout the period of orthodontic tooth movement (14 days). Further research of the changes in alveolar bone and underlying periodontal tissue that occur following led exposure over time during orthodontic tooth movement may be necessary to understand better the biologic mechanisms that occur.

\section{Conclusions}

The exposure of LED increases collagen density on the pressure and tension side of the periodontal ligament as compared to the control group. The observation day of the pressure side decreased on day 3 and increased on day 7 up to day 14. However, the density increased from day 3 onwards on the tension side. It is suggested that further studies determine other cells affected by exposure to LED in the alveolar bone remodeling process as a way to develop this research for clinical application. Otherwise, further study can compare the length of exposure and the observation period of blue Light Emitting Diode to the collagen density in the pressure and tension of periodontal ligament sides of orthodontic tooth movement after 14 days.

\section{Funding}

Not applicable.

\section{Acknowledgments}

Not applicable.

\section{Conflicts of Interest}

The authors have no conflicts of interest regarding this investigation.

\section{References}

1. Suparwitri, S.; Christnawati; Retnaningrum, Y.; Alhasyimi, A.A. Effects of Administering the Soybean Isoflavone Genistein on Alkaline Phosphatase Levels During Orthodontic Tooth Movement in Young and Old Rabbits. Arch. Orofac. Sci 2021, 16, 33-40, https://doi.org/10.21315/aos2021.16.1.4.

2. Arianda, T.A.; Rezqita, P.; Pudyani, P.S.; Rosyida, N.F.; Alhasyimi A.A. Effect of cocoa administration on osteoblast counts and alkaline phosphatase levels during orthodontic tooth movement in rats. J Orofac Sci. 2021, 12, 101-106, https://doi.org/10.4103/jofs.jofs_51_20.

3. Alhasyimi, A.A.; Rosyida, N.F. Cocoa administration may accelerate orthodontic tooth movement by inducing osteoclastogenesis in rats. Iran $J$ Basic Med Sci 2020, 22, 206-10, https://doi.org/10.22038/ijbms.2018.32967.7881.

4. Dungel, P.; Hartinger, J.; Chaudary, S.; Slezak, P.; Hofmann, A.; Hausner, T.; et al. LLLT by LED of different wavelength induces angiogenesis and improves ischemic wound healing. Lasers Surg Med 2014, 46, 773-80, https://doi.org/10.1002/1sm.22299. 
5. Yasseai, S.; Fekrazad, R.; Shahraki, N. Effect of Low Level Laser Therapy on Orthodontic Tooth Movement: A Review Article. Journal of Dentistry of Tehran University of Medical Sciences 2013, 10, 264-72, https://www.ncbi.nlm.nih.gov/pmc/articles/PMC4264099/.

6. Li, Y.; Jacox, L.A.; Little, S.H.; Ko, C.C. Orthodontic Tooth Movement: The biology and clinical implications, Kaohsiung. J Med Sci. 2018, 34, 207-14, https://doi.org/10.1016/j.kjms.2018.01.007.

7. Khalimatun, S.S.; Mohamad, R.J.; Elysha, N.I.; Reezal, I. The effect of Nigella sativa and Eucheuma cottonii in Collagen-induced Arthritis mice. Research J. Pharm. and Tech 2020, 13, 1319-23, https://doi.org/10.5958/0974-360X.2020.00243.7.

8. Ekizer, A.; Uysal, T.; Guray, E. Effect of LED-mediated Photobiomodulation Therapy on Orthodontic Tooth Movement and Root Resorption in Rats. Laser Med Sci. 2013, 30, 779-85, https://doi.org/10.1007/s10103013-1405-3.

9. Armellin, E.; Bovessecchi, G.; Coppa, P.; Pasquantonio, G.; Cerronil, L. LED Curing Lights and Temperature Changes in Different Tooth Sites. Biomed Res. Int 2016, 18, 4672-82, https://doi.org/10.1155/2016/1894672.

10. Zein, R.; Selting, W.; Hamblin; Michael, R. Review of light parameters and photobiomodulation efficacy: dive into complexity. J Biomedical Optics 2018, 23, 120901, https://doi.org/10.1117/1.JBO.23.12.120901. .

11. Rahmah, N.E.; Christnawati; Suparwitri, S. The Effect of Blue-Light Emitting Diode Irradiation to Alkaline Phosphatase Levels of Orthodontic Tooth Movement. J Int Dent Med Res 2020, 13, 1242-7, https://www.semanticscholar.org/paper/The-Effect-of-Blue-Light-Emitting-Diode-Irradiation-RahmahChristnawati/f793c3f83e152232750e0efbad7cb5f153e4527c.

12. Friedrichsdorf, S.P.; Chavez, V.E.A.; Bradaschia-Correa, V.; Cattaneo, P.M.; Dominguez, G.C. Infrared Light-Emitting Diode (LED) Effects on Orthodontic Tooth Movement. Braz Dent J. 2020, 30, 410-6, https://doi.org/10.1590/0103-6440201902416.

13. Hamblin, M.R. Mechanisms and Applications of The Anti-Inflammatory Effects of Photobiomodulation. AIMS Biophys 2017, 4, 337-361, https://dx.doi.org/10.3934\%2Fbiophy.2017.3.337.

14. Tam, S,Y.; Tam, V.C,W.; Ramkumar, S.; Khaw, M.L.; Law, H.K.W.; Lee, S.W.Y. Review on the Cellular Mechanisms of Low-Level Laser Therapy Use in Oncology. Front Oncol. 2020, 10, 1255, https://doi.org/10.3389/fonc.2020.01255.

15. Rajendran, N.K.; George, B.P.; Chandran, R.; Tynga, I.M.; Houreld, N.; Abrahamse, H.; The Influence of Light on Reactive Oxygen Species and NF-кB in Disease Progression. Antioxidants 2020, 8, 640, https://doi.org/10.3390/antiox8120640.

16. De Lima, F.M.; Moreira, L.M.; Villaverde, A.B.; Albertini, R.; Castro-Faria-Neto, H.C.; Aimbire, F. Lowlevel laser therapy (LLLT) acts as cAMP-elevating agent in acute respiratory distress syndrome. Lasers Med. Sci. 2011, 26, 389-400, https://doi.org/10.1007/s10103-010-0874-x.

17. Ishihara, A.; Park, I.; Suzuki, Y. et al. Metabolic responses to polychromatic LED and OLED light at night. Sci Rep. 2021, 11, 12402, https://doi.org/10.1038/s41598-021-91828-6.

18. Suparwitri, S.; Rosyida, N.F.; Alhasyimi, A.A. Wheat seeds can delay orthodontic tooth movement by blocking osteoclastogenesis in rats. Clin. Cosmet. Investig. Dent. 2020, 11, 243-9, https://doi.org/10.2147/CCIDE.S212886.

19. Prayogo, R.D.; Sandy, B.N.; Sujarwo, H.; Fitri, K.; Brahmanta, A.; Rahardjo, P.; Handayani, B. The Changes of Fibroblast and Periodontal Ligament Characteristics in Orthodontic Tooth Movement with Adjuvant HBOT and Propolis: A study in Guinea pigs. Padjadjaran. J Dent 2020, 32, 48-56, https://doi.org/10.24198/pjd.vol32no1.19848.

20. Li, Y.; Zhan, Q.; Bao, M.; et al. Biomechanical and biological responses of periodontium in orthodontic tooth movement: up-date in a new decade. Int J Oral Sci 2021, 13, 20, https://doi.org/10.1038/s41368-021-001255.

21. Alhasyimi, A.A.; Rosyida, N.F.; Rihadini, M.S. Postorthodontic relapse prevention by administration of grape seed (vitis vinifera) extract containing cyanidine in rats. Eur J Dent 2020, 13, 629-34, https://doi.org/10.1055/s-0039-3401440.

22. Alhasyimi, A.A.; Suparwitri, S.; Christnawati. Effect of Carbonate Apatite Hydrogel-Advanced Platelet-Rich Fibrin Injection on Osteoblastogenesis during Orthodontic Relapse in Rabbits. Eur J Dent. 2021, 15, 412-9, https://doi.org/10.1055/s-0040-1721234. 